\title{
Current Status and Challenges of Endoscopic Treatments for Duodenal Tumors
}

\author{
Yasutoshi Ochiai $^{\mathrm{a}}$ Motohiko Kato $^{\mathrm{a}}$ Yoshiyuki Kiguchi $^{\mathrm{a}}$ Teppei Akimoto $^{\mathrm{a}}$ \\ Atsushi Nakayama $^{a}$ Motoki Sasaki $^{\mathrm{a}}$ Ai Fujimoto $^{\mathrm{a}, \mathrm{b}}$ Tadateru Maehata $^{\mathrm{a}}$ \\ Osamu Goto ${ }^{a, c}$ Naohisa Yahagi ${ }^{a}$ \\ a Division of Research and Development for Minimally Invasive Treatment, Cancer Center, Keio University School \\ of Medicine, Tokyo, Japan; b Division of Gastroenterology, National Hospital Organization Tokyo Medical Center, \\ Tokyo, Japan; ' Gastroenterology and Hepatology, Nippon Medical School, Tokyo, Japan
}

\author{
Keywords \\ Duodenal tumor · Endoscopic submucosal dissection . \\ Complication
}

\begin{abstract}
Background: Superficial tumors of the duodenum, other than ampullary tumors, have been traditionally considered rare. However, reports of this kind of tumor have increased in recent times, and the demand for minimally invasive treatments have also increasing. Summary: Adenomas and intramucosal carcinomas are target lesions for treatment. A preoperative diagnosis has not been established, but unnecessary biopsies increase the difficulty of treatment and should be avoided. Cold snare polypectomy, endoscopic mucosal resection (EMR), and underwater EMR are treatment options for small lesions. Endoscopic submucosal dissection enables secure resection even for large lesions, but it is technically demanding and associated with a higher complication rate. After endoscopic resections, exposure of digestive juices is believed to cause delayed complications. To prevent these complications, several closing and covering methods are proposed, with favorable clinical results. Key message: A treatment method should be chosen based
\end{abstract}

\section{KARGER}

๑ 2018 S. Karger AG, Basel

E-Mail karger@karger.com

www.karger.com/dig on each patient's situation. With any of the treatment methods, post-resection measures are required to prevent delayed complications. Secure closure of resection wounds seems promising.

(c) 2018 S. Karger AG, Basel

\section{Introduction}

Superficial tumors of the duodenum, with the exception of ampullary tumors, have traditionally been considered rare and not highly malignant. According to previous reports, the incidence in autopsied cases is $0.02-0.5 \%$ [1-3]. Of these cases, primary duodenal carcinomas have been reported to comprise approximately $0.5 \%$ of malignant tumors of the gastrointestinal tract [4]. However, recent progress in endoscopic equipment, improvements in endoscopic techniques, and increased awareness have gradually increased the number of reported primary duodenal carcinomas. According to a multi-center survey by Goda et al. [5], the number of superficial non-ampullary duodenal epithelial tumors (SNADETs) resected endoscopically or surgically in the 6-year period between 2007 and 2012 (396 lesions, 364 cases) increased by over 2 -fold 
in a short time, that is, from 125 lesions in the first half of this period to 271 cases in the second half of this period. Surgical treatments for duodenal tumors, such as pancreaticoduodenectomy, are known to be highly invasive and associated with high morbidity. Thus, less invasive treatments such as endoscopic mucosal resection (EMR) and endoscopic submucosal dissection (ESD) would be a more optimal treatment option for adenomas and intramucosal carcinomas, similar to superficial tumors arising from other parts of the gastrointestinal tract. However, organ specificity and treatment difficulty have interfered with the standardization of endoscopic treatments of large SNADETs. Moreover, because of their low incidence, there is limited data regarding duodenal tumors. Herein, we describe the current status and challenges associated with endoscopic treatment for SNADETs based on experiences at our institution.

\section{Target Lesions}

Although the characteristics of SNADETs are unclear, previous studies have reported that among early-stage cancers, intramucosal carcinomas without vascular infiltration have a low risk of metastasis; thus, local treatment by endoscopic resection is feasible [5]. Furthermore, the adenoma-carcinoma sequence is believed to be one of the pathways in the carcinogenesis of duodenal carcinomas, and data from our experiences suggest adenomas as the target for treatment, given that the carcinoma-bearing rate increases with the enlargement of tumor diameter. Various investigations have been conducted regarding a preoperative diagnosis. Endo et al. [6] have attempted to use crystal violet staining for SNADETs, as for colorectal lesions, and classified SNADETs into convoluted, leaflike, reticular/sulciolar, and colon-like patterns using conventional colorectal diagnostic endoscopy techniques. Furthermore, Yoshimura et al. [7] have investigated superficial microstructural findings using magnifying endoscopy combined with narrow-band imaging. They reported that the heterogeneous pattern was observed in all cases, but the obscure pattern was found significantly more frequently in adenomas with high-grade dysplasia and intramucosal carcinomas. In addition, they classified mucosal microvascular structures to have (1) a network pattern and (2) an intra-villous-structure irregular pattern and reported that the network pattern was notable in adenomas with high-grade dysplasia and intramucosal carcinoma, particularly in depressed types. However, the number of cases are limited in both retro- spective studies, and an accurate modality for a differential diagnosis between adenomas and early-stage carcinoma by endoscopy has yet to be established [8]. Regarding the biopsy of SNADETs, Kinoshita et al. [9] reported a sensitivity of $37.5 \%$, specificity of $83.1 \%$, and accuracy of $71.6 \%$, which are far from sufficient levels. Moreover, a biopsy should be prevented, as it may cause submucosal fibrosis, leading to a non-lifting situation with submucosal injection, thereby increasing the difficulty of endoscopic treatment (Fig. 1). Therefore, whether to use endoscopic treatment should be determined on a case-bycase basis depending on the size and location of the tumor as well as maneuverability of the endoscope being considered. Given that a preoperative diagnosis is not established yet, endoscopic resection techniques enabling enbloc resection should be selected with the assumption that a more precise histopathological diagnosis is available with en-bloc resection than piece-meal resection.

\section{Treatment Methods}

In general, EMR is performed on small SNADETs since it is easy and fast with a low complication rate. Thus, SNADETs approximately less than $1 \mathrm{~cm}$ are reported to be good targets for this technique, although this may vary depending on the situation [10]. Recently, duodenal cold snare polypectomy has been attempted for small SNADETs less than $1 \mathrm{~cm}$ [11]. It is an easy technique and is expected to reduce the risk of delayed perforation or bleeding because there is no thermal damage induced by the high-frequency current. However, similar to the colon, it is difficult to secure a lateral margin, thereby raising a concern of incomplete resection. Presently, multi-center prospective studies are being conducted to evaluate its safety and efficacy. For 1-2 cm SNADETs, EMR is still the first choice endoscopic treatment; however, it is sometimes difficult to obtain successful results due to insufficient lifting after the submucosal injection. Underwater EMR [12-14], originally described by Binmoeller et al. [14], is a unique technique that snares and resects the target lesion under water without lifting by local injection. Since the intestinal tract is not stretched by insufflation, capturing target lesions up to $2 \mathrm{~cm}$ in size within water is expected to be easier even in the duodenum and should result in a successful outcome [12-14]. To prevent major invasive surgeries such as pancreaticoduodenectomy, ESD can be performed for large lesions that are unresectable by other endoscopic resection techniques. However, the technical difficulties and risk of complications are ex- 

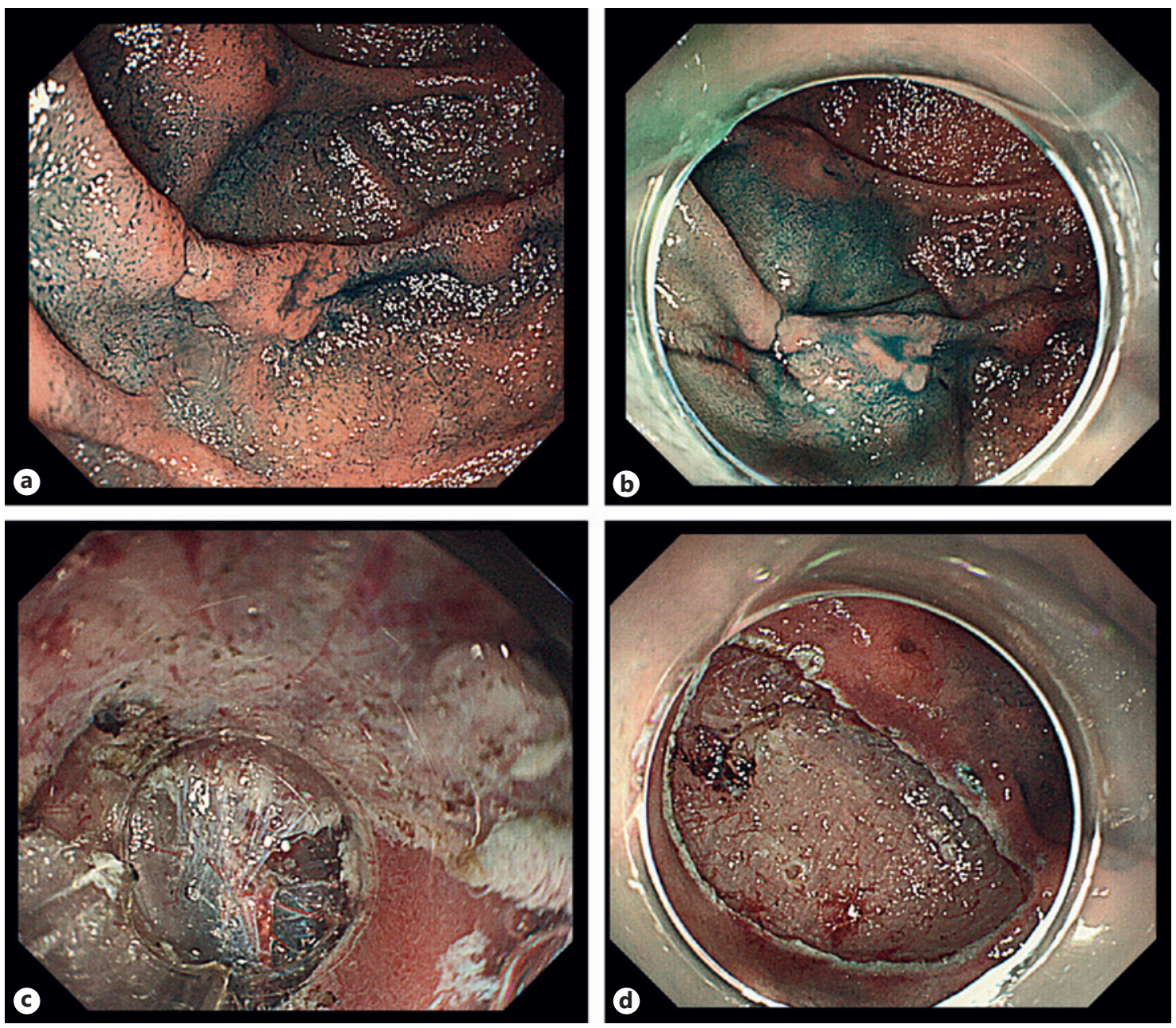

Fig. 1. Duodenal flat lesion with fibrosis caused by biopsy. a A small flat lesion was found in the descending part of the duodenum. Endoscopic mucosal resection was planned. b Remarkable non-lifting sign was found after submucosal injection. c Fibrous submucosa was dissected by endoscopic submucosal dissection. d Complete resection was achieved without any complication. tremely high in duodenal ESD, requiring this procedure to be performed only by experts in specialized institutions [15-16]. In addition to the above-mentioned endoscopic resection techniques, laparoscopic and endoscopic cooperative surgery (LECS), which was originally developed for the treatment of submucosal tumors of the stomach [15], is attempted for SNADETs with local resection or securing closure of the resection wound by laparoscopy after ESD $[17,18]$. Although it reduces the risks of postoperative complications, LECS has several challenges. Basically, there is a potential risk of tumor cell dissemination to the abdominal cavity when local resection is performed, since it exposes the tumor's surface to the abdominal cavity [19]. Furthermore, LECS cannot be performed in areas where it is difficult to undertake the laparoscopic approach (e.g., the area attached to pancreas head), and caution is required when performing LECS for large lesions due to the risk of postoperative stricture (Table 1).

\section{Problems of Duodenal ESD}

In Japan, ESD is widely performed as a standard endoscopic treatment in most of the gastrointestinal organs, except for the duodenum. It is not widely performed in the duodenum because of the difficulties in handling the endoscope. The duodenum is the deepest point at which a normal upper GI endoscope can reach and is situated be- 
Table 1. Treatment method according to tumor size

\begin{tabular}{|c|c|c|c|}
\hline Treatment method & Pros & Cons & Indication \\
\hline Cold polypectomy & Safe, convenient & Local residue & $\begin{array}{l}\text { Lesion size < } 10 \mathrm{~mm} \text { (i.e., } \\
\text { lesion associated with FAP) }\end{array}$ \\
\hline EMR/UEMR & Relatively safe & $\begin{array}{l}\text { Inferior } \mathrm{R} 0 \text { resection rate when } \\
\text { compared with ESD }\end{array}$ & Lesion size $<20 \mathrm{~mm}$ \\
\hline $\begin{array}{l}\text { Partial duodenectomy } \\
\text { (including D-LECS }{ }^{\#} \text { ) }\end{array}$ & $\begin{array}{l}\text { Less invasive when compared } \\
\text { with pancreaticoduodenectomy } \\
\text { Secure suturing after resection }\end{array}$ & $\begin{array}{l}\text { Deformity or stricture for wide field } \\
\text { resection } \\
\text { Inferior curability when compared } \\
\text { with pancreaticoduodenectomy }\end{array}$ & $\begin{array}{l}\text { Lesion size } 20-40 \mathrm{~mm}^{*} \\
\text { Endoscopically unresectable }\end{array}$ \\
\hline Pancreaticoduodenectomy & High curability & High invasiveness & $\begin{array}{l}\text { Any size lesion with } \\
\text { suspicion of invasive cancer } \\
\text { Endoscopically unresectable }\end{array}$ \\
\hline
\end{tabular}

yond the pyloric ring. Thus, the endoscope can bend in the stomach even after it has been stretched once, making it difficult to achieve good endoscopic control in many cases. Moreover, injected fluid tends to spread along the fold, making it difficult to create good lifting. In addition, as the proper muscle layer is extremely thin, pressing the knife to the target tissue can often cause perforation during the procedure. Additionally, sometimes the sharp edges of metallic clips cause secondary perforation when we attempt clip closure of the perforated area. Lastly, if we leave the post-ESD wound open, the risk of subsequent bleeding or delayed perforation becomes extremely high in the duodenum due to exposure to pancreatic juice and bile [20]

\section{Ingenuities of ESD in Duodenum}

As mentioned above, duodenal ESD is technically very challenging. Several ingenuities and modifications of the ESD technique have been proposed to overcome these difficulties. We reported a water pressure method in which water irrigation is used just after a mucosal incision to open the submucosal space [21]. By irrigating normal saline from the endoscope with the tip of the small-caliber-tip transparent hood (ST Hood, Fujifilm Co., Ltd., Tokyo, Japan) tightly contacted to the incision edge, the water pressure opens the incision line for initial submu- cosal dissection under direct visualization to ensure safe entry into the submucosal layer. Once in the submucosal layer, the subsequent submucosal dissection becomes relatively easy and safe. Miura et al. [22] also reported the pocket-creation method, which involves making a pocket in the submucosal layer with a minimal initial mucosal incision after sufficient submucosal injection. In this technique, the tip of the endoscope becomes stable within the pocket, which facilitates the subsequent submucosal dissection. These modified ESD techniques are expected to improve the outcomes of duodenal ESD.

\section{Techniques to Prevent Complications}

Exposure to digestive juices is believed to cause postoperative complications of duodenal ESD. To prevent these complications, several closing and covering methods have been reported. As such, the string clip suturing method [23] was developed for closing a relatively large resection wound after ESD which seemed unable to be closed with standard clips alone. Unlike the previously reported double-channel endoscope and loop snare method [24], this simple method enables closure of the resection wound by using a single channel endoscope and a string tied to a clip [25] (Fig. 2). Another measure of closure is the over-the scope clip (OTSC ${ }^{\circledR}$, Ovesco Endos- 

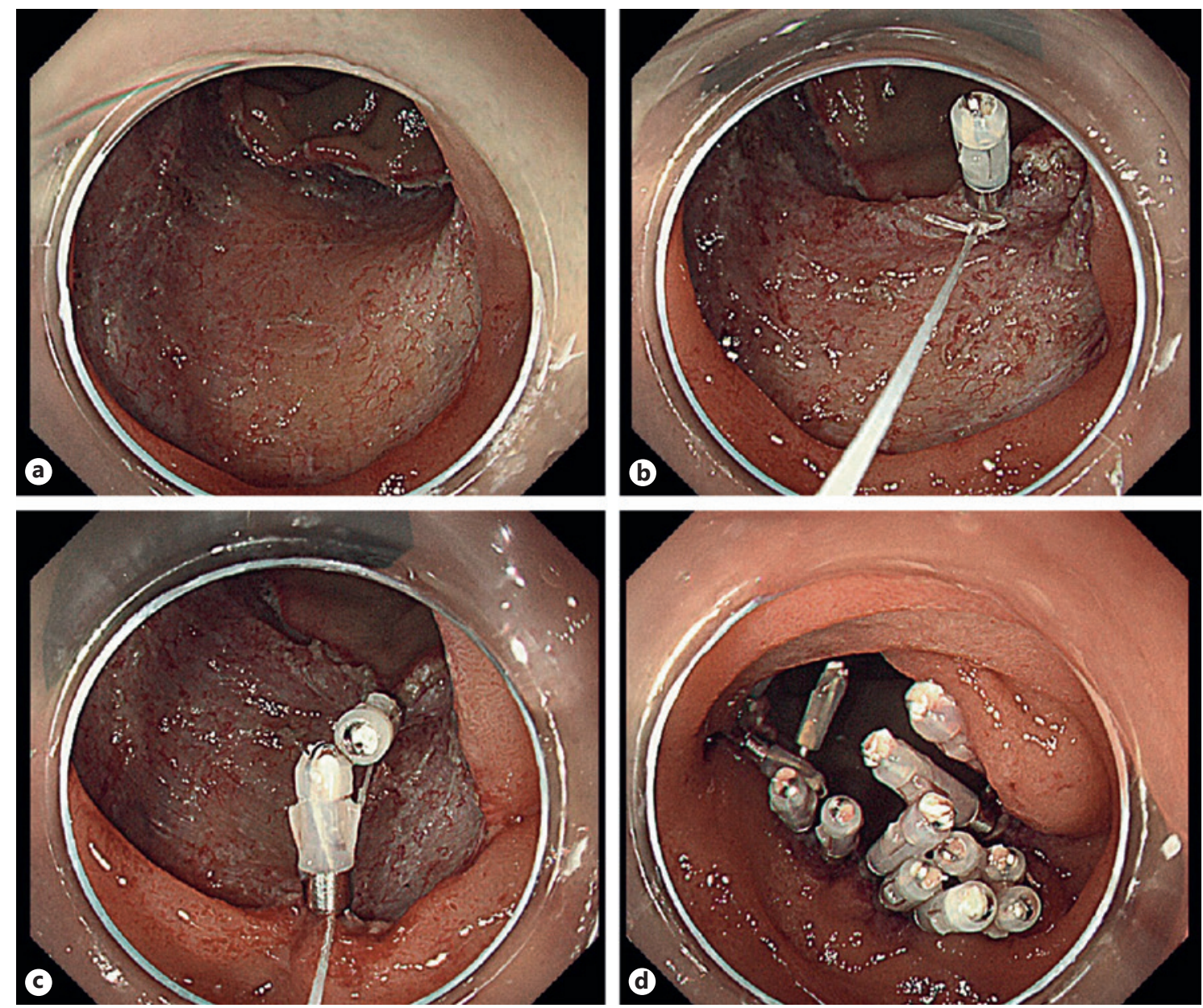

Fig. 2. String clip suturing method. a The large mucosal defect after endoscopic submucosal dissection, measuring approximately $5 \mathrm{~cm}$. b A clip with a string was attached on the distal edge of the resection bed. c The string was also anchored on the proximal edge of the resection bed. Subsequently, the large resection bed was approximated by pulling the string before applying additional clips. d Finally, the large resection bed was completely closed with additional clips. copy AG, Tübingen, Germany). It is a large endoscopic clip mounted onto the tip of the endoscope with an applicator. Surrounding healthy mucosa around the resection wound can be tucked into the OTSC by suction, similar to endoscopic variceal ligation, or it can be pulled in by grasping forceps. This technique is supposed to be promising for securing closure of mucosal defects after endoscopic treatment, especially in the duodenum [26]. However, high cost for the device still remains an issue. Covering the mucosal defect with a polyglycolic acid sheet is an alternative option. Takimoto et al. [27] reported a covering method using polyglycolic acid sheets delivered through a working channel of an endoscope and pasted with fibrin glue. With either method, the complication rate is decreased when the resection wound is properly closed or covered; hence, they are considered crucial additional procedures of duodenal $\operatorname{ESD}[15,28]$. Additionally, in cases where closing or covering a resection wound by those techniques proves difficult, placing an endoscopic naso-pancreatic drainage tube and an endoscopic naso-biliary drainage tube can be effective in preventing delayed complications, and this approach prevents exposure to digestive juices.

\section{Conclusions}

Although diagnostic endoscopy of the duodenum is not yet established, biopsy should be avoided because of its low accuracy and the risk of developing fibrosis. Cold 
snare polypectomy, EMR, and underwater EMR are suitable and feasible methods for treating small lesions. Although ESD is still technically challenging and even risky, it is effective for selected targets including large lesions. Since the delayed complication rate is relatively high in the duodenum, it requires post-resection measures to prevent delayed complications. Secure closure of the resection wound seems promising.

\section{Ethics Statement}

The authors have no ethical conflicts to disclose.

\section{Disclosure Statement}

The authors declare no conflicts of interest associated with this manuscript.

\section{References}

1 Darling RC, Welch CE: Tumors of the small intestine. N Engl J Med 1959;260:397-408.

2 Hoffman BP, Grayzel DM: Benign tumors of the duodenum. Am J Surg 1945;70:394-400.

3 Shukla SK, Elias EG: Primary neoplasms of the duodenum. Surg Gynecol Obstet 1976; 142:858-860.

4 Alwmark A, Andersson A, Lasson A: Primary carcinoma of the duodenum. Ann Surg 1980; 191:13-18.

5 Goda K, Kikuchi D, Yamamoto Y, Takimoto K, Kakushima N, Morita Y, et al: Endoscopic diagnosis of superficial non-ampullary duodenal epithelial tumors in japan: multicenter case series. Dig Endosc 2014;26 (suppl 2):2329.

6 Endo M, Abiko Y, Oana S, Kudara N, Chiba T, Suzuki K, et al: Usefulness of endoscopic treatment for duodenal adenoma. Dig Endosc 2010;22:360-365.

7 Yoshimura N, Goda K, Tajiri H, Ikegami M, Nakayoshi T, Kaise M: Endoscopic features of nonampullary duodenal tumors with narrowband imaging. Hepatogastroenterology 2010; 57:462-467.

8 Tsuji S, Doyama H, Tsuji K, Tsuyama S, Tominaga $\mathrm{K}$, Yoshida N, et al: Preoperative endoscopic diagnosis of superficial non-ampullary duodenal epithelial tumors, including magnifying endoscopy. World J Gastroenterol 2015; 21:11832-11841.

9 Kinoshita S, Nishizawa T, Ochiai Y, Uraoka T, Akimoto T, Fujimoto A, et al: Accuracy of biopsy for the preoperative diagnosis of superficial nonampullary duodenal adenocarcinoma. Gastrointest Endosc 2017;86:329-332.

10 Yamasaki Y, Uedo N, Takeuchi Y, Ishihara R, Okada H, Iishi H: Current status of endoscopic resection for superficial nonampullary duodenal epithelial tumors. Digestion 2018;97: $45-51$.

11 Maruoka D, Matsumura T, Kasamatsu S, Ishigami H, Taida T, Okimoto K, et al: Cold pol- ypectomy for duodenal adenomas: a prospective clinical trial. Endoscopy 2017;49:776783.

12 Yamasaki Y, Uedo N, Takeuchi Y, Higashino $\mathrm{K}$, Hanaoka N, Akasaka T, et al: Underwater endoscopic mucosal resection for superficial nonampullary duodenal adenomas. Endoscopy 2018;50:154-158.

13 Kato M, Tsujii Y, Takehara T: Underwater endoscopic mucosal resection of a duodenal adenoma with biopsy scars. Dig Endosc 2018;30: 405-416.

14 Binmoeller KF, Shah JN, Bhat YM, Kane SD: "Underwater" emr of sporadic laterally spreading nonampullary duodenal adenomas (with video). Gastrointest Endosc 2013;78: 496-502.

15 Hoteya S, Furuhata T, Takahito T, Fukuma Y, Suzuki Y, Kikuchi D, et al: Endoscopic submucosal dissection and endoscopic mucosal resection for non-ampullary superficial duodenal tumor. Digestion 2017;95:36-42.

16 Yahagi N, Kato M, Ochiai Y, Maehata T, Sasaki M, Kiguchi Y, et al: Outcomes of endoscopic resection for superficial duodenal epithelial neoplasia. Gastrointest Endosc 2018; 88:676-682.

17 Hiki N, Yamamoto Y, Fukunaga T, Yamaguchi T, Nunobe S, Tokunaga M, et al: Laparoscopic and endoscopic cooperative surgery for gastrointestinal stromal tumor dissection. Surg Endosc 2008;22:1729-1735.

18 Otowa Y, Kanaji S, Morita Y, Suzuki S, Yamamoto M, Matsuda Y, et al: Safe management of laparoscopic endoscopic cooperative surgery for superficial non-ampullary duodenal epithelial tumors. Endosc Int Open 2017;5: E1153-E1158.

19 Goto O, Shimoda M, Sasaki M, Kiguchi Y, Mitsunaga Y, Akimoto T, et al: Potential for peritoneal cancer cell seeding in endoscopic full-thickness resection for early gastric cancer. Gastrointest Endosc 2018;87:450-456.
20 Fujihara S, Mori H, Kobara H, Nishiyama N, Matsunaga T, Ayaki M, et al: Management of a large mucosal defect after duodenal endoscopic resection. World J Gastroenterol 2016; 22:6595-6609.

21 Yahagi N, Nishizawa T, Sasaki M, Ochiai Y, Uraoka T: Water pressure method for duodenal endoscopic submucosal dissection. Endoscopy 2017;49:E227-E228.

22 Miura Y, Shinozaki S, Hayashi Y, Sakamoto H, Lefor AK, Yamamoto H: Duodenal endoscopic submucosal dissection is feasible using the pocket-creation method. Endoscopy. 2017;49:8-14.

23 Yahagi N, Nishizawa T, Akimoto T, Ochiai Y, Goto O: New endoscopic suturing method: String clip suturing method. Gastrointest Endosc 2016;84:1064-1075.

24 Akimoto T, Goto O, Nishizawa T, Yahagi N: Endoscopic closure after intraluminal surgery. Dig Endosc 2017;29:547-558.

25 Kato M, Ochiai Y, Fukuhara S, Maehata T, Sasaki M, Kiguchi Y, et al: Clinical impact of closure of the mucosal defect after duodenal endoscopic submucosal dissection. Gastrointest Endosc 2018:pii:S0016-5107(18)32867-0.

26 Tashima T, Ohata K, Sakai E, Misumi Y, Takita M, Minato Y, et al: Efficacy of an over-thescope clip for preventing adverse events after duodenal endoscopic submucosal dissection: a prospective interventional study. Endoscopy $2018 ; 50: 487-496$.

27 Takimoto K, Imai Y, Matsuyama K: Endoscopic tissue shielding method with polyglycolic acid sheets and fibrin glue to prevent delayed perforation after duodenal endoscopic submucosal dissection. Dig Endosc 2014;26 (suppl 2):46-49.

28 Nishizawa T, Akimoto T, Uraoka T, Mitsunaga Y, Maehata T, Ochiai Y, et al: Endoscopic string clip suturing method: a prospective pilot study (with video). Gastrointest Endosc 2018;87:1074-1078. 